\title{
Plasmon and Grid Resonances in the Electromagnetic Scattering by Finite Grids of Silver Nanowires
}

\author{
Denys M. Natarov ${ }^{1, *}$, Trevor M. Benson, ${ }^{2}$ Ayhan Altintas, ${ }^{3}$ Ronan Sauleau ${ }^{4}$, Alexander I. Nosich ${ }^{1,5}$ \\ ${ }^{I}$ Institute of Radio-Physics and Electronics NASU, Proskury 12, 61085, Kharkiv, Ukraine \\ Tel: +380-(57)-720-3782, Fax: +380-(57)-315-2105 \\ ${ }^{2}$ George Green Institute for Electromagnetics Research, University of Nottingham, NG7 2RD, UK \\ ${ }^{3}$ Bilkent University, Ankara 06800, Turkey \\ ${ }^{4}$ IETR, Université de Rennes 1, Rennes Cedex 35042, France \\ ${ }^{5}$ Université Européenne de Bretagne, c/o Université de Rennes 1, Rennes Cedex 35042, France \\ *Corresponding author: den.natarov@gmail.com
}

\begin{abstract}
The problem of the H-polarized wave scattering by finite chains of circular nanowires is considered. A twodimensional diffraction problem with rigorous boundary conditions is solved by partial separations of variables method using local polar coordinates of each scatterer. The obtained results demonstrate convergence of the algorithm and good agreement with data known for the conducting and dielectric cylinders. Plasmonic and grid resonances are found and calculated for grids from a big number of silver nanowires. This opens a way to the accurate numerical simulation of various finite configurations of wires met in today's nano and microsize photonic devices.
\end{abstract}

Keywords: nanowires, plasmon and grid resonances, total scattering cross section, absorption scattering cross section.

\section{INTRODUCTION}

In this paper we study two kinds of resonance scattering of light by structured nanosize scatterers. The first is the plasmon or electrostatic resonance. It is known that small material objects can exhibit resonance behavior at certain frequencies for which the object permittivity is negative and the free-space wavelength is large in comparison with object dimensions. The latter condition clearly suggests that these resonances are electrostatic in nature. They appear at specific negative values of dielectric permittivity (eigenvalues) for which source-free electrostatic fields may exist. The physical mechanism behind these resonances is the presence of free electron gas in noble metal particles that may display collective oscillations and give the major contribution to the dielectric permittivity at optical frequencies. For nanoscale metallic objects, plasmon resonances result in powerful localized sources of scattered light that are useful in the scanning near-field optical microscopy, nanolithography, and biosensor applications. It is also believed that strong local electromagnetic fields associated with these resonances may play an important role in the surface Raman scattering. Currently, such resonances are found experimentally or numerically by probing dielectric objects of complex shapes with radiation of various frequencies [1]. Here, important result has been obtained in [2]. It has been found that the plasmon-resonance frequencies can be directly computed as the (real-negative) eigenvalues of permittivity for a specific boundary integral equation. Given the dispersion of permittivity, this brings the quasistatic resonances. Thus, plasmon resonances have unique physical property: in the leading terms, resonance frequencies depend on the object shape but do not depend on its geometric dimensions. For example, they may shift considerably if the noble-metal wires are collected in closely spaced assemblies.

The second kind of resonances is observed in periodical structures: this is the grating resonance. Wood was the first who discovered multiple drastic transformations of the scattering pattern and reflectance intensity if changing the light frequency or the angle of incidence [3]. One of such "anomalies," first theoretically explained by Lord Rayleigh is observed if one of the Floquet harmonics is "passing over horizon" [4]. In the simplest case of the normal incidence, the Rayleigh-Wood anomalies are observed if the period of the grating is multiple to the wavelength. When solving a problem of diffraction by a finite grating, for example, by a grid of $M$ wires, one cannot reduce it to one period and must treat the grid as an $M$-body scatterer [5]. It is quite interesting to find out what is the effect of periodicity on the scattering by finite grids, and how large the number $M$ should be to consider a wire grid as "physically infinite." As a criterion, one can choose the appearance of sharp lobes in the far-field scattering pattern in the directions of the Floquet-modes of infinite grid; however it is difficult to quantify this effect. Still besides of the Rayleigh anomaly, among other Wood anomalies in the infinite-grating scattering one can see the resonances on the "grating modes," whose frequencies are just below the Rayleigh frequencies if the wires are a fraction of the period [6]. Therefore in this paper we study the formation of such resonance in the scattering of the H-polarized plane waves by the large enough finite grids of sub-wavelength silver wires. 


\section{FORMULATION AND BASIC EQUATIONS}

We consider the two dimensional (2-D) scattering of the plane wave $\left(\sim e^{-i \omega t}\right)$ by finite chains of equidistantly located wires as shown in Fig. 1. The wires are modelled as infinite circular cylinders with the same radius $a$ and relative dielectric permittivity $\varepsilon$. The distance between adjacent cylinders is $\rho$ and their number is $M$.

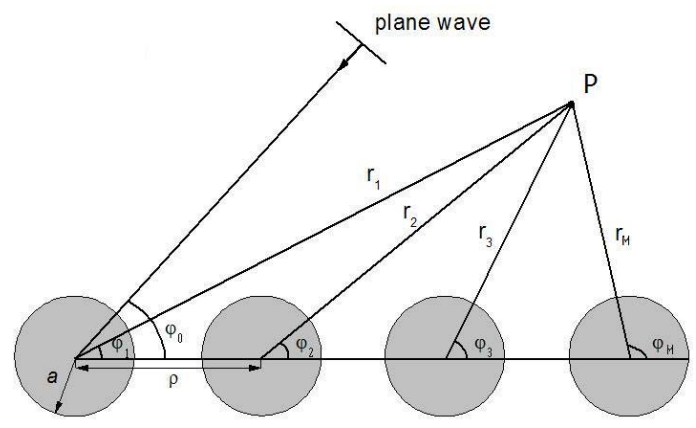

Figure 1. Scattering problem and notations.

The global coordinates have the origin at the centre of the first cylinder. For a 2-D problem, a scalar function $U$, which represents either $E_{z}$ or $H_{z}$ scattered-field component, must satisfy the Helmholtz equation with wavenumber $k_{-}=\sqrt{\varepsilon} k_{0}$ or $k_{+}=k_{0}$ inside and outside of each cylinder, the total tangential field continuity conditions, the radiation condition at infinity, and the condition of the local power finiteness. The solution can be obtained by expanding the field function in terms of the azimuth exponents in the local coordinates (Fig. 1), using addition theorems for cylindrical functions, and applying the boundary conditions on all $M$ cylinders.

The unknown coefficients related to the $q$-th cylinder include the effect of all interactions between the cylinders [5,7]. They satisfy an infinite matrix equation, $(\mathbf{I}+\mathbf{U}) \mathbf{X}=\mathbf{U}^{0}$, where $\mathbf{U}=\left\{\delta_{i j} U^{(i, j)}\right\}_{i, j=1, \ldots M}$, $\mathbf{X}=\left(x_{m}^{(1)} \ldots x_{m}^{(M)}\right), \quad \mathbf{U}^{0}=\left(U_{m}^{0(1)} \ldots U_{m}^{0(M)}\right), i, j=1, \ldots M$, and $m=0, \pm 1, \ldots$. This is $M \times M$ block-type Fredholm second kind equation, where each block is infinite. Therefore the solution of corresponding counterpart equation with each block truncated to finite order $N$ converges when the number $N$ gets greater. To characterize far-field scattering properties of considered grids we have used the total scattering cross section (TCS) and absorption scattering cross section (ACS) frequency dependences. We have tested our code and got a good agreement with the results of [8-10].

\section{NUMERICAL RESULTS AND DISCUSSION}

In computations, we have considered the scattering of the H-polarized plane waves by finite grids of parallel silver nanowires with radii about tens of nanometers, in the visible band. Note that for particles or wires in the range of tens of nanometers one can use the bulk experimental data obtained by Johnson and Christie [11] to characterize the complex dielectric permittivity of silver.

In Fig. 2, are presented the wavelength dependences of TCS and ACS normalized to $M$ for the grids of $M=800,1600$ and 3200 silver nanowires illuminated by the normally incident H-wave in the visible band.

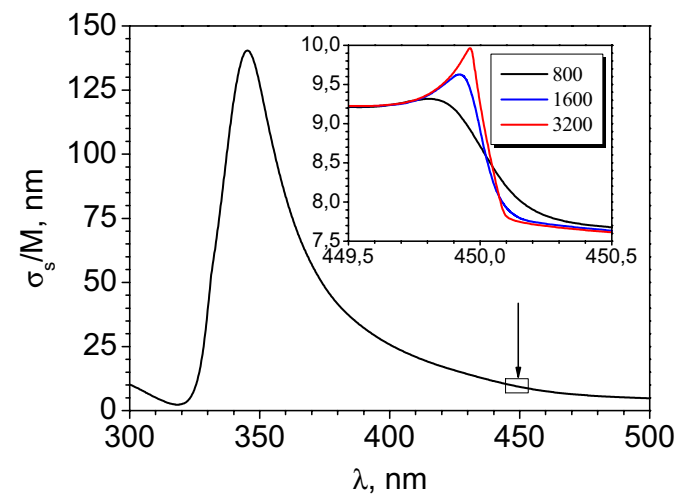

(a)

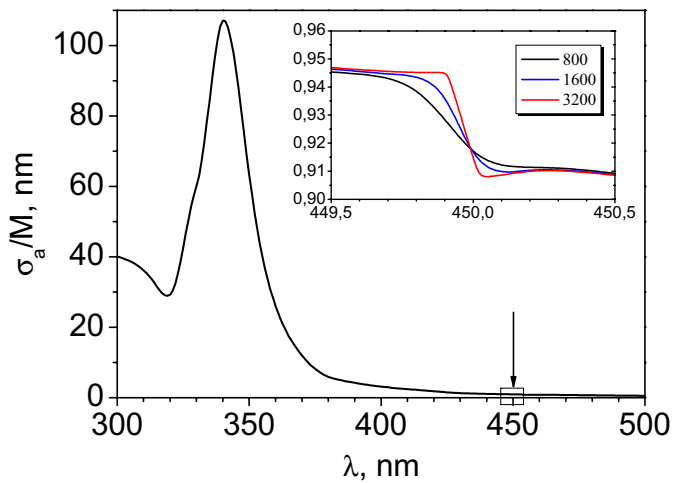

(b)

Figure 2. Normalized total (a) and absorption (b) scattering cross sections as a function of wavelength for the $H$-wave incident on the different grids of silver nanowires with radii $a=25 \mathrm{~nm}$ and period $\rho=450 \mathrm{~nm}$. 
The radius of each cylinder is $a=25 \mathrm{~nm}$ and the distance between their centers (i.e. grid period) is $\rho=450 \mathrm{~nm}$. One can see a broad resonance at $\lambda=345.2 \mathrm{~nm}$. This is the plasmon resonance [10], which has the same wavelength and shape as for a stand-alone silver wire because the distance between adjacent cylinders is too large for quasistatic effects.

Besides of the plasmon resonance, both scattering cross sections have their value leaps in the vicinity of the wavelength $450 \mathrm{~nm}$, which corresponds to the distance between the grid element centers. This smaller resonance amplitude increases (Fig. 2a) with the number of scatterers in the grid.

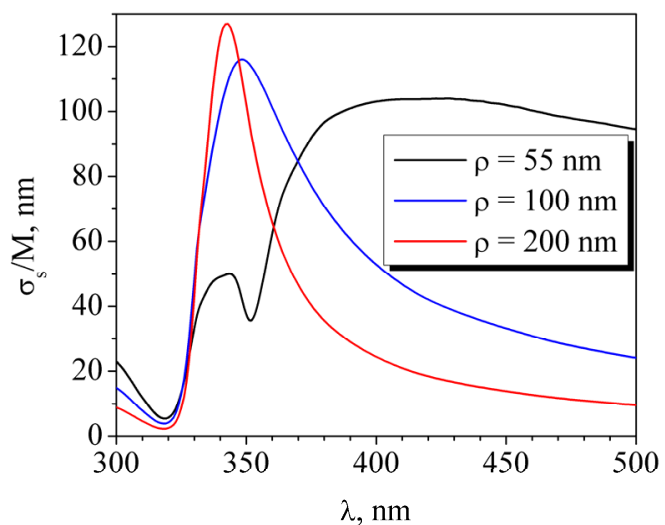

(a)

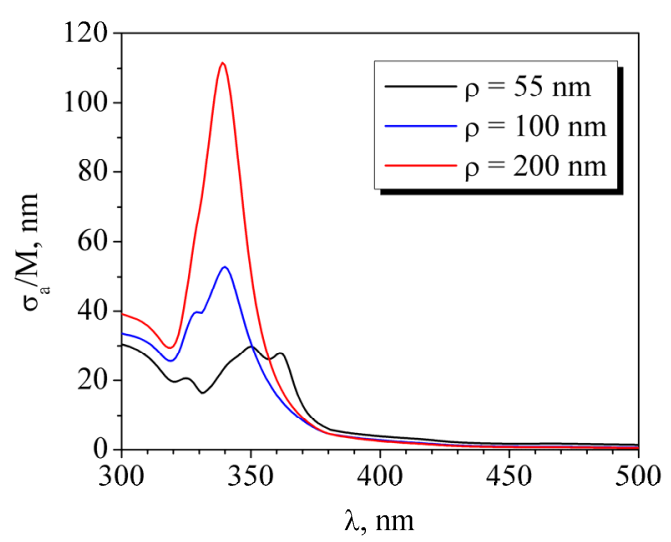

(b)

Figure 3. The same as in Fig. 2 for the grid of 400 silver nanowires with different $\rho$ and radii a $=25 \mathrm{~nm}$.

In Fig. 3, the same values are presented for a grid of 400 cylinders of radius $a=25 \mathrm{~nm}$ for three distances between their centers: $\rho=55 \mathrm{~nm}, 100 \mathrm{~nm}$ and $200 \mathrm{~nm}$. In the case of the $5 \mathrm{~nm}$ distance between adjacent their influence on each other is very impressive. One can see that the first resonance peak in TSC for $\rho=55 \mathrm{~nm}$ has lower values than for a single cylinder and ASC has a local minimum at this resonance wavelength and a maximum where TSC reaches a local minimum. In Fig. 3a one can see the second very broad TSC resonance for $\rho=55 \mathrm{~nm}$. These resonances are associated with different charge configurations on the scatterer surfaces. The field in the gap between adjacent cylinders has extremely big values at the resonance frequencies. The value and width of plasmon resonances depends of distance between adjacent cylinders and their radius.

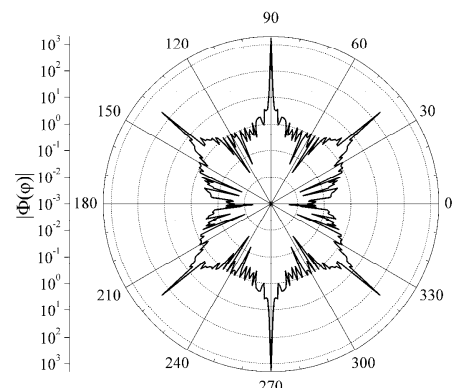

(a)

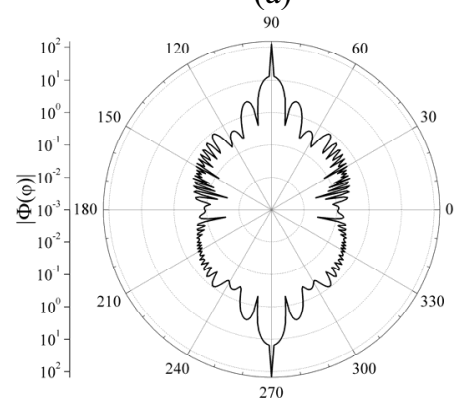

(c)

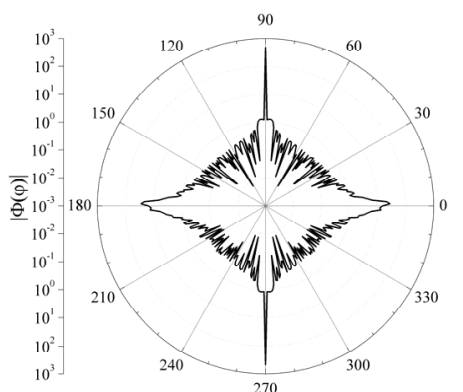

(b)

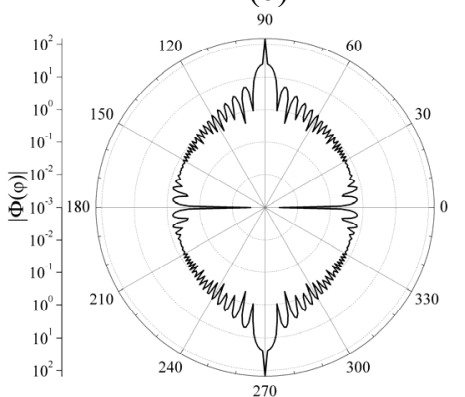

(d)

Figure 4. Far-field scattering patterns for the H-wave incident normally on the 1600 silver nanowire grid with period $\rho=450 \mathrm{~nm}$ in the plasmon (a) and grating (b)resonances, and on the 400 nanowire grid with $\rho=55 \mathrm{~nm}$ in the plasmon resonance (c) and off resonance (d); $a=25 \mathrm{~nm}$ 
In Fig. 3 one can see the variation of TSC and ASC resonances if the grid period increases. As the coupling of elements to each other decreases, the TSC and ASC dependences tend to the same dependences for a stand-alone silver nanowire. This curve can be also observed in Fig. 2; it coincides with the data of [10] obtained by completely different albeit convergent and accurate method.

In Fig. 4a and Fig. 4b are presented the far-field scattering patterns for the grids of 1600 silver nanowires with radius $a=25 \mathrm{~nm}$ and period $\rho=450 \mathrm{~nm}$ at the plasmon-resonance wavelength $\lambda=345 \mathrm{~nm}$ (Fig. $4 \mathrm{a})$ and at the grating- resonance wavelength $\lambda=449.9 \mathrm{~nm}$ (Fig. 4b). In Fig. 4c and Fig. 4d are presented the far-field scattering patterns for the grids of 400 silver nanowires with $\rho=55 \mathrm{~nm}$ at the plasmon-resonance wavelength $\lambda=$ $344 \mathrm{~nm}$ (Fig. 4c) and at the non-resonant for this configuration wavelength $\lambda=450 \mathrm{~nm}$ (Fig. 4d). They have narrow main lobes looking in $\varphi=\pi / 2$ (specular reflection lobe) and $3 \pi / 2$ (shadow lobe) directions. In addition, the pattern in Fig. 4a has four sharp lobes corresponding to the propagation angles of the \pm 1 -st Floquet harmonics in the infinite grid, because in this case wavelength $(345 \mathrm{~nm})$ is smaller than grating period $(450 \mathrm{~nm})$. In the case of the grid-mode resonance (Fig. 4b) one can see large pattern values in both lateral directions $(\varphi=0$ and $\pi$ ), however in the other figures the scattering in the lateral directions is small.

\section{CONCLUSIONS}

We have presented accurate results for the scattering and absorption cross-sectrions for the H-polarized plane wave diffraction by finite linear grids of silver nanowires with the numbers of elements up to 3200. A 2-D diffraction problem with rigorous boundary conditions has been treated by the partial separation of variables using local polar coordinates of each scatterer. This has enabled us to reduce it to a Fredholm second kind blockmatrix equation with favorable features. We have demonstrated two different kinds of resonances: plasmon and grid-type, and studied how they depend on the number of nanosize silver wires in the periodically structured linear grid of wires. The plasmon resonance keeps the same shape and frequency as for a stand-alone wire so far as the air gap between adjacent wires is not smaller than the wire diameter. It clearly dominates in the frequency scans of TCS and ACS if the number of wires is 3200 or less. However, if this number gets larger, a narrow peak associated with the grid-type resonance on the wavelength very close to the grid period may be expected. Another interesting observation, which seems to escape the attention of researchers earlier, is that plasmonresonance values of TCS and ACS are remarkably close to each other.

\section{ACKNOWLEDGEMENTS}

The authors are grateful to E.I. Smotrova and I.V. Ivanchenko for many helpful discussions. This work has been partially supported by the Ministry of Education and Science, Ukraine by the project M/146-2010, the Royal Society, UK via joint project IJP-2007/R1, and the TUBITAK and NASU via exchange project EEEAG106E209.

\section{REFERENCES}

[1] V. Giannini, J. A. Sanchez-Gil: Calculations of light scattering from isolated and interacting metallic nanowires of arbitrary cross section by means of Green's theorem surface integral equations in parametric form, J. Optical Society of America, vol. 24, no 9, pp. 2822 - 2830, 2007.

[2] D. R. Fredkin, I. Mayergoyz: Resonant behavior of dielectric objects (electrostatic resonances), Physical Review Letters, vol. 91, no 25, pp. 253902-1 - 253902-4, 2003.

[3] R. W. Wood: On a remarkable case of uneven distribution of light in a diffraction grating spectrum, Philosophical Magazine, vol. 4, pp. 396-403, 1902.

[4] Lord Rayleigh: On the dynamical theory of gratings, Proc. Royal Soc. London, vol. A-79, pp. 399-416, 1907.

[5] V. Twersky: Multiple scattering of radiation by an arbitrary planar configuration of parallel cylinders, J. Acoust. Soc. Am., vol. 24, pp. 407-414, 1952.

[6] R. Gomez-Medina, M. Laroche, J. J. Saenz: Extraordinary optical reflection from sub-wavelength cylinder arrays, Optics Express, vol. 14, no 9, pp. 3730-3737, 2006.

[7] A. Z. Elsherbeni: A comparative study of two-dimensional multiple scattering techniques, Radio Science, vol. 29, no. 4, pp. 1023-1033, 1994.

[8] G. O. Olaofe: Scattering by two cylinders, Radio Science, vol. 4, no. 11, pp. 1351 - 1360, 1970.

[9] H. A. Ragheb, M. Hamid: Scattering by N parallel conducting circular cylinders, Int. J. Electronics, vol. 59, no 4, pp. 407-421, 1985.

[10] J. P. Kottman, O. J. F. Martin: Plasmon resonant coupling in metallic nanowires, Optics Express, vol. 8, no. 12 , pp. 655-663, 2001.

[11] R. W. Christy, P. B. Johnson: Optical constants of the noble metals, Physical Review B, vol. 6, no 12, pp. 4370-4378, 1972. 